\title{
LA VISIÓN DEL CONCEPTO DE FEMICIDIO EN EL PROFESORADO DE MATEMÁTICA. UN ESTUDIO DE CASO
}

A VISÃO DO CONCEITO DE FEMICÍDIO NO PROFESSORADO DE
MATEMÁTICA. UM ESTUDO DE CASO

Verónica Molfino

Consejo de Formación en Educación (CFE) Uruguay

Cristina Ochoviet

Consejo de Formación en Educación (CFE) Uruguay

Daniela Pagés

Consejo de Formación en Educación (CFE) Uruguay

\begin{abstract}
Resumen
En este trabajo analizamos una secuencia de enseñanza para la formación de profesores, que aborda el concepto de femicidio y que fue diseñada desde la perspectiva de la enseñanza de la matemática para la justicia social por parte de una profesora de matemática, cursando estudios de posgrado. La producción de la docente revela la manera en que entiende el femicidio y discutimos su visión cotejando con la figura jurídica de este concepto, establecida en Uruguay por la Ley Actos de discriminación y femicidio. El estudio realizado ofrece evidencia de que la profesora parecería confundir homicidio femenino con femicidio y que esta visión se ve reflejada en la secuencia de enseñanza. Esto podría llevar a los destinatarios de la secuencia, futuros profesores de matemática, a la construcción de una visión errónea de esta figura social y jurídica.
\end{abstract}

Palabras clave: Formación de professores. Femicidio. Justicia social.

\section{Resumo}

Neste trabalho analisamos uma sequência de ensinamento para a formação de professores, que aborda o conceito de 


\section{LA VISIÓN DEL CONCEPTO DE FEMICIDIO EN EL PROFESORADO DE MATEMÁTICA. UN ESTUDIO DE CASO}

feminicídio e que foi desenhada desde a perspectiva do ensino de matemática para a justiça social por parte de uma professora de matemática, cursando pós-graduação. $\mathrm{O}$ trabalho da docente revela a maneira que entende o feminicídio e discutimos sua visão, confrontado com a figura jurídica deste conceito, estabelecida em Uruguai pela Lei atos de discriminação e feminicídio. O estudo realizado oferece evidência de que a professora parecia confundir homicídio feminino com feminicídio e que essa visão se vê refletida na sequência do ensino. Isso poderia levar os destinatários da sequência, futuros professores de matemática, a formação de uma visão errônea desta figura social e jurídica.

Palavras-chave: Formação de professores. Feminicídio. Justiça social.

\section{Introducción}

La enseñanza de la matemática para la justicia social (EMpJS) es una perspectiva que surge a partir de la consideración de la matemática y de su enseñanza como actividades netamente humanas, no neutrales, en cuyo proceso de construcción intervienen intereses de diversos grupos sociales. Por otra parte, implica la consideración de que es posible leer, comprender y escribir el mundo empleando recursos matemáticos (GUTSTEIN, 2006; SKOVMOSE, 2012).

Este trabajo tiene por objetivo reportar la visión del concepto de femicidio de una profesora de matemática, cursando estudios de postgraduación en modalidad virtual, que emergió al solicitársele que diseñara una secuencia de enseñanza desde la perspectiva de la EMpJS, para ser aplicada en la formación de profesores de matemática. En particular, nos propusimos identificar qué visión del concepto de femicidio queda en evidencia a través de la secuencia didáctica elaborada por la profesora.

Este estudio adquiere especial importancia pues constituye un ejemplo de cómo una noción incorrecta acerca de un constructo social puede interferir en la formación de futuros profesores, y por consiguiente, en la ciudadanía en su conjunto. En concreto, la visión de la profesora sobre femicidio podría conducir a la construcción de visiones incorrectas del concepto por parte de los futuros profesores y, a su vez, el riesgo podría trasladarse a alumnos de enseñanza media.

\section{Antecedentes}



MATEMÁTICA. UN ESTUDIO DE CASO

Los antecedentes temáticos están organizados en dos secciones. En la primera presentamos trabajos realizados por la comunidad internacional en torno a la EMpJS y en la segunda abordamos los trabajos realizados en Uruguay desde este punto de vista.

\section{La enseñanza de la matemática para la justicia social en la formación docente}

Si bien la EMpJS se presenta con diferencias en sus significados, el concepto de enseñanza para la justicia social aparece cada vez con más énfasis en los programas de formación docente (STINSON; BIDWELL; POWELL, 2012, siguiendo a MCDONALD, 2007), en el entendido de la necesidad de preparar a los futuros profesores para la diversidad o multiculturalidad. Este desarrollo se aprecia particularmente en Estados Unidos y Gran Bretaña.

La EMpJS persigue dos tipos de objetivos: promover la justicia social en el ámbito del aula, atendiendo a la vez a la construcción de conocimiento matemático (GUTSTEIN, 2006). Tratar de cumplir estos objetivos implica grandes desafíos (FELTON-KOESTLER, 2017; STINSON; BIDWELL; POWELL, 2012).

Algunos trabajos señalan la resistencia de los futuros profesores (AGUIRRE, 2009; ENSIGN, 2005; FELTON; SIMIC-MULLER; MENÉNDEZ, 2012; RODRÍGUEZ, 2005, citados por FELTON-KOESTLER, 2017). Rodríguez (2005) señala resistencias de los estudiantes de profesorado a enseñar considerando inclusivamente la culturalidad de los estudiantes (diversidad en el plano ideológico). También hace referencia a la resistencia a enseñar hacia la comprensión, a través de aproximaciones constructivistas, aprendizaje basado en la indagación y usando abordajes intelectualmente estimulantes para los futuros profesores (diversidad pedagógica).

Otro grupo de investigaciones que reporta Felton-Koestler (2017) plantean que el enfoque de la perspectiva de la EMpJS lleva a que esta sea más aceptada, a la vez que los futuros profesores la comprenden más profundamente (ENSIGN, 2005; FELTON; KOESTLER, 2012, 2015; FELTON; SIMIC-MULLER; MENÉNDEZ, 2012).

Tenemos, entonces, desde la investigación en el nivel internacional, trabajos que señalan la importancia de reflexionar sobre el desafío que conlleva, para futuros profesores y para sus formadores, conectar los conceptos matemáticos, la atención a los aspectos culturales y el abordaje de problemáticas referidas a la justicia social. 


\title{
LA VISIÓN DEL CONCEPTO DE FEMICIDIO EN EL PROFESORADO DE MATEMÁTICA. UN ESTUDIO DE CASO
}

\section{Enseñanza de la matemática para la justicia social en Uruguay}

Reportamos a continuación los trabajos que se vienen desarrollando en Uruguay desde 2016.

En primer lugar, presentamos una investigación reportada en dos artículos (LÓPEZ; GUERRA, 2017; GUERRA; LIM; LÓPEZ, 2017), en la que comparan las primeras respuestas de futuros docentes de primaria en relación con la inclusión de la EMpJS en su formación. Esta comparación se realiza entre dos instituciones en contextos distintos, social y culturalmente: una universidad de Estados Unidos y una institución de formación docente de Uruguay.

\begin{abstract}
Las autoras comparan el grado de aceptación de este enfoque entre los dos grupos, la que es mayor entre los estudiantes uruguayos. En efecto, estos señalan interés por este enfoque para la enseñanza de la matemática y buena disposición para profundizar en esta perspectiva (LÓPEZ; GUERRA, 2017). Los futuros maestros estadounidenses, en tanto, no consideran que este enfoque sea apropiado, ya que no es responsabilidad de los docentes la enseñanza de la justicia social. Además, manifiestan temor a castigos o incluso al despido, por trabajar temáticas que no están en el currículo. Estos dos tipos de reacciones también son reportadas por Felton-Koestler (2017).
\end{abstract}

Desde 2016 se han realizado un conjunto de trabajos entre formadores de profesores, estudiantes de profesorado de matemática de una institución de formación de Montevideo y docentes de matemática de enseñanza secundaria. Estos estudios se enfocan en el diseño de secuencias de enseñanza que promueven la EMpJS, a través de la implementación de distintos recursos didácticos. Entre estos, se utilizaron noticias de prensa (LEIRÓS; RAMÍREZ; OCHOVIET, 2016), narración oral de cuentos (DOLGAY; OCHOVIET, 2016; SCHAFFEL; OCHOVIET, 2016), situaciones personales (GONZÁLEZ; GONZÁLEZ; LEPRATTE; MOLFINO; VIERA, 2016), datos del mercado (DE LEÓN; DELGADO; MOLFINO; SANTINI, 2016; ÁLVAREZ; MOLFINO; PEREIRA; SILVA, 2017), teatro de títeres (BENTANCORT et al., 2017), estudios socioestadísticos (GALLI; MONTEGUI; MOLFINO; NÚÑEZ, 2017; MOLFINO; PERDOMO; RUIZ; VILLA, 2017). Otro trabajo fue diseñado para una clase de álgebra lineal en la formación de profesores de matemática, con base en un texto literario (Un mundo feliz de Aldous Huxley) como recurso pedagógico (COLOMBO, 2017). Las propuestas de algunos de estos trabajos fueron llevadas al aula, y se analizaron en 

MATEMÁTICA. UN ESTUDIO DE CASO

forma colaborativa. Molfino y Ochoviet (2019) reportan las dificultades de un grupo de profesores para comprender la perspectiva de la EMpJS y para elaborar actividades de aprendizaje enfocadas en ella, en el marco de una asignatura de sus estudios de postgraduación.

Estos estudios abordan dos de las perspectivas de EMpJS señaladas por FeltonKoestler (2017): conectar la matemática con la identidad social y cultural de los estudiantes y usar la matemática para el desarrollo de la conciencia crítica y el trabajo para cambiar las injusticias en nuestra sociedad.

En este trabajo realizamos un abordaje novedoso, no estudiado anteriormente, que consiste en analizar de qué manera la conceptualización de una profesora de matemática de un constructo social y jurídico, en este caso el femicidio, incide en un diseño didáctico para la enseñanza de la matemática.

\section{Perspectiva teórica}

\section{La enseñanza de la matemática para la justicia social}

Los inicios de la pedagogía crítica son asociados a los trabajos que Paulo Freire desarrolló a partir de 1970 sobre alfabetización y pedagogía de la liberación (STINSON; BIDWELL; POWELL, 2012). Varios autores vinculan estos trabajos con los fundamentos de la EMpJS (GONZÁLEZ, 2009; GUTSTEIN, 2006; LÓPEZ; GUERRA, 2017; WRIGHT, 2014).

Felton-Koestler (2017) afirma que los trabajos con enfoque en la EMpJS comienzan a desarrollarse incipientemente en la última década del siglo XX y cobran impulso en la primera quincena del siglo XXI. Según este autor las discusiones en torno a la EMpJS y la educación matemática crítica abarcan muy diversas perspectivas que, no obstante, tienen en común la consideración de dos conjuntos de objetivos pedagógicos dialécticamente relacionados: uno relativo a la justicia social y el otro a la matemática (GUTSTEIN, 2006). 


\section{LA VISIÓN DEL CONCEPTO DE FEMICIDIO EN EL PROFESORADO DE MATEMÁTICA. UN ESTUDIO DE CASO}

Metas sobre justicia social según Gutstein (2006)

\begin{tabular}{|c|c|}
\hline \multicolumn{2}{|c|}{ Metas sobre justicia social según Gutstein (2006) } \\
\hline $\begin{array}{c}\text { Leer el mundo con } \\
\text { matemática }\end{array}$ & $\begin{array}{c}\text { Entender las relaciones de poder. } \\
\text { Entender las desigualdades de } \\
\text { recursos y oportunidades. } \\
\text { Entender la discriminación } \\
\text { explícita entre diferentes grupos } \\
\text { sociales basada en raza, clase, } \\
\text { género, lenguaje y otras } \\
\text { diferencias. }\end{array}$ \\
\hline $\begin{array}{c}\text { Escribir el mundo con } \\
\text { matemática }\end{array}$ & $\begin{array}{c}\text { Reescribir el mundo para } \\
\text { cambiarlo. }\end{array}$ \\
\hline $\begin{array}{c}\text { Desarrollar identidades } \\
\text { culturales y sociales } \\
\text { positivas }\end{array}$ & $\begin{array}{c}\text { Instruir matemática en el lenguaje, } \\
\text { cultura y comunidad de los } \\
\end{array}$ \\
estudiantes, brindándoles el \\
conocimiento matemático \\
necesario para sobrevivir y \\
prosperar en la cultura dominante.
\end{tabular}

Tabla 1. Metas sobre justicia social propuestas por Gutstein (2006) y su significado según Stinson, Bidwell y Powell (2012).

Fuente: Elaboración propia.

\begin{tabular}{|c|c|}
\hline \multicolumn{2}{|c|}{$\begin{array}{c}\text { Metas pedagógicas relativas a la matemática según } \\
\text { Gutstein (2006) }\end{array}$} \\
\hline Leer el mundo matemático & $\begin{array}{c}\text { Desarrollar estrategias de } \\
\text { generalización. } \\
\text { Resolver en forma creativa } \\
\text { problemas no rutinarios. } \\
\text { Percibir a la matemática como } \\
\text { herramienta para la crítica } \\
\text { sociopolítica. }\end{array}$ \\
\hline $\begin{array}{c}\text { Tener éxito académico en } \\
\text { el sentido tradicional }\end{array}$ & $\begin{array}{c}\text { Aprobar pruebas estandarizadas, } \\
\text { avanzar en los estudios, acceder } \\
\text { a cursos matemáticos de mayor } \\
\text { nivel o a carreras relacionadas } \\
\text { con la matemática cuando el } \\
\text { estudiante lo elige. }\end{array}$ \\
\hline $\begin{array}{c}\text { Cambiar la concepción de } \\
\text { estudiantes y profesores } \\
\text { sobre la matemática }\end{array}$ & $\begin{array}{c}\text { herramienta poderosa de } \\
\text { análisis para entender } \\
\text { problemas complejos del mundo } \\
\text { real. }\end{array}$ \\
\hline
\end{tabular}

Tabla 2. Metas pedagógicas relativas a la matemática y su significado según Gutstein (2006).

Fuente: Elaboración propia. 
LA VISIÓN DEL CONCEPTO DE FEMICIDIO EN EL PROFESORADO DE MATEMÁTICA. UN ESTUDIO DE CASO

Como muchos autores señalan, introducir la perspectiva de la EMpJS es un gran desafío, especialmente en programas de formación de profesores (FELTON-KOESTLER, 2017; AGUIRRE, 2009; ENSIGN, 2005).

\section{El marco Qué, Quién y Cómo de la matemática}

Felton-Koestler (2017) introduce el marco Qué, Quién y Cómo (WWH por su sigla en inglés) para comprender la visión de los profesores y su propia instrucción como docentes del proceso de enseñanza. Este marco permite informar acerca de la enseñanza de la matemática, considerando las creencias de los profesores y la naturaleza de la matemática escolar.

La EMpJS tiene un claro foco en la sociedad o el medio en el que vivimos y por ello, el WWH se concentra en las relaciones entre la matemática que se enseña en las instituciones educativas y el mundo en el que las instituciones están insertas, entendiendo por mundo, o también mundo real, el contexto sociopolítico de la institución de referencia.

Felton-Koestler (2017, p. 50-51)) se centra en tres preguntas clave:

- ¿Qué mensajes da la enseñanza de la matemática?

- ¿De quiénes son las perspectivas que deberían estar representadas en la matemática escolar y de qué manera se relacionan con los estudiantes?

- ¿Cómo deberían estar relacionados los conceptos matemáticos con el mundo real en la matemática escolar?

Según el autor estas preguntas son lo suficientemente amplias como para permitir poner la atención en una variedad de situaciones educativas.

En este estudio nos focalizamos en la primera de las preguntas, en concreto: ¿Qué mensajes da acerca de la noción de femicidio la enseñanza de matemática que propone una profesora?

Las otras dos preguntas las utilizaremos para elaborar recomendaciones a partir del presente estudio. 


\section{LA VISIÓN DEL CONCEPTO DE FEMICIDIO EN EL PROFESORADO DE MATEMÁTICA. UN ESTUDIO DE CASO}

\section{Método}

En el marco del curso de posgrado "Aportes metodológicos para la enseñanza de la Matemática en la formación de profesores de Matemática", dictado en línea en un aula Moodle, propusimos a los participantes (seis profesores de matemática cursando estudios de postgraduación) la siguiente actividad:

(1) Leer cuatro documentos de referencia específicos de EMpJS y educación matemática crítica (LLORENTE, 2012, SKOVSMOSE, 2012, PARRA, 2013, WRIGHT, 2014).

(2) Discutir en foro, a partir de preguntas elaboradas por las docentes del curso, las ideas de Llorente (2012) y Wright (2014).

(3) Diseñar una actividad y su implementación en un grupo del profesorado de matemática (en la asignatura que se considere conveniente) adoptando como enfoque la EMpJS y teniendo como meta a lograr por parte de los estudiantes de profesorado: "leer el mundo a partir de recursos matemáticos" (SKOVSMOSE, 2012, p. 65).

De los seis profesores participantes, solo una abordó el concepto de femicidio para el diseño de la secuencia de enseñanza. Es este caso el que constituye el foco de este estudio.

Para alcanzar el objetivo (identificar qué visión del concepto de femicidio queda en evidencia a través de la secuencia didáctica elaborada por la profesora), nos basamos en la producción de la participante que consistió en el diseño de una secuencia de enseñanza desde la perspectiva de la EMpJS que favoreciera la lectura del mundo a partir de recursos matemáticos. Es del análisis de esta producción que obtendremos pistas para develar la visión de la docente acerca del concepto de femicidio.

\section{Análisis de la secuencia de enseñanza}

La profesora diseñó la siguiente actividad:

Lea el siguiente artículo http://www.lr21.com.uy/comunidad/1386535-femicidioshomicidios-mujeres-violencia-genero-ministerio-interior-bonomi

1. Realiza una tabla de frecuencias (frecuencia absoluta, frecuencia relativa, porcentaje) con los datos sobre las conexiones y reconexiones de pulseras electrónicas en 2018. Representa los datos en una gráfica circular, sabiendo que la población uruguaya en ese año es de 3460181.

2. Determina el tipo de variable que es la cantidad de llamadas diarias denunciando violencia doméstica y similares, justifica. 


\section{LA VISIÓN DEL CONCEPTO DE FEMICIDIO EN EL PROFESORADO DE MATEMÁTICA. UN ESTUDIO DE CASO}

3. Investiga la diferencia entre femicidio y homicidio. ¿Cuál crees que sea la relación entre la tasa de homicidios a mujeres y la tasa de homicidios a hombres? Ingresa en el siguiente

link

https://www.minterior.gub.uy/genero/images/stories/Femicidios_Uruguay.pdf

4. En la página 22, compara la tasa de homicidios a mujeres y varones ¿qué opinión merecen los datos?

5. Realiza la misma comparación con los mapas que se presentan en las páginas 2728.

6. Selecciona una gráfica del capítulo 3 y realiza su estudio.

7. ¿En qué departamento crees que la tasa de femicidio cometido por la (ex)pareja es mayor? ¿Y en cuál es la menor? ¿Por qué?

a. Compara tus respuestas con la segunda tabla de la página 78 .

b. ¿Cuál es la probabilidad de que un hombre de Salto haya cometido homicidio a su (ex)pareja desde los años 1996 hasta 2016?

8. Considerando la tabla de la página 78, donde se muestran los años y la cantidad de homicidios a mujeres por parte de su (ex)pareja (HMP) y los datos del artículo de La Red 21:

a. Describe su comportamiento.

b. Si pudieras predecir ¿cómo será el comportamiento en los próximos años? ¿Por qué?

El primer artículo seleccionado por la docente para la actividad no refiere directamente al tema del femicidio, sino a homicidios a mujeres, y se focaliza en el porcentaje de estos cuya causa fue la violencia doméstica. Este artículo es utilizado por la profesora para las tres primeras partes de la actividad. Encontramos que las dos primeras de ellas (realizar una tabla de frecuencias sobre la conexión y reconexión de tobilleras, indicar qué tipo de variable es el número de llamadas de denuncias por violencia doméstica y similares) no parecen muy conectadas entre sí ni con el concepto de femicidio, que aparece hasta aquí confundido con el de homicidio a una mujer. En la tercera parte, la actividad propone investigar la diferencia entre femicidio y homicidio. Sin embargo, en la consigna de comparar sus tasas, vuelve a hablar de homicidio a mujeres en lugar de femicidio. Y en la fundamentación de esta parte de la actividad, la profesora plantea como objetivo, que "los estudiantes entiendan y discutan la diferencia entre homicidio y femicidio, y que no hay ninguna palabra que se utilice para referirse únicamente al homicidio de hombres". Agrega que aquí puede discutirse o profundizarse sobre "si esto es por el feminismo" o "va en contra de la igualdad de género". La profesora también señala que le llama la atención que la tasa de homicidios a hombres es mayor que la de homicidios a mujeres, y se plantea analizar las ideas previas de los estudiantes en relación con esto.

De la actividad planteada, así como de la fundamentación de la docente, interpretamos que tiene un concepto equivocado de femicidio, pues considera como tal cualquier homicidio 


\title{
LA VISIÓN DEL CONCEPTO DE FEMICIDIO EN EL PROFESORADO DE MATEMÁTICA. UN ESTUDIO DE CASO
}

a una mujer, o tal vez aquellos que resultan de la violencia doméstica. En Uruguay existe, desde 2002, la Ley de erradicación de la violencia doméstica. Esta establece que:

\begin{abstract}
Constituye violencia doméstica toda acción u omisión, directa o indirecta, que por cualquier medio menoscabe, limitando ilegítimamente el libre ejercicio o goce de los derechos humanos de una persona, causada por otra con la cual tenga o haya tenido una relación de noviazgo o con la cual tenga o haya tenido una relación afectiva basada en la cohabitación y originada por parentesco, por matrimonio o por unión de hecho. (Ley 17514, Art. 2).
\end{abstract}

En el contexto de esta ley se considera violencia doméstica a la ejercida sobre cualquier persona. El concepto de femicidio aparece en la Ley 19538 de 2017, que agrega al Código Penal la figura de femicidio, homicidio "contra una mujer por motivos de odio, desprecio o menosprecio, por su condición de tal” (Ley 19538, Art. 3).

Esta confusión que parece tener la profesora aparece a lo largo de la actividad que propone. Si bien en el análisis de la actividad presenta definiciones correctas de femicidio y de homicidio, como ya dijimos, trata al femicidio como sinónimo de homicidio a mujeres.

El segundo documento que la docente propone para la actividad, titulado Femicidios íntimos en Uruguay, sí se centra en los femicidios, específicamente los cometidos por la (ex) pareja de la víctima. Sin embargo, en las partes 4 y 5 de la actividad, la docente centra nuevamente la propuesta en la comparación entre homicidios a mujeres y homicidios a hombres.

En la fundamentación a esta parte de la actividad, la profesora escribe:

... se puede destacar que en los países nombrados la tasa de homicidios a hombres es mayor a la tasa de homicidios a mujeres. En la puesta en común se puede preguntar ¿por qué si la tasa de homicidios a hombres es mayor se le da tanta importancia al femicidio? ¿Qué concepción de las mujeres existe en Uruguay?

Estas preguntas que la profesora propone para los estudiantes sugieren que ella misma considera que el femicidio no es un problema en sí mismo, ya que la tasa de homicidio a hombres es mayor que el cometido sobre mujeres. No logra identificar que si hay un nombre específico para el crimen femicidio y no para el correspondiente sobre los hombres, es porque no existe como tal.

Las partes 7 y 8 de la actividad parecen centrarse en el femicidio, aunque, al igual que el resto de la actividad, la temática aparece más como una excusa para tratar los conceptos 


\section{LA VISIÓN DEL CONCEPTO DE FEMICIDIO EN EL PROFESORADO DE MATEMÁTICA. UN ESTUDIO DE CASO}

matemáticos que la docente se propone trabajar, que como una oportunidad de "leer el mundo a partir de recursos matemáticos". El hecho de que no presente preguntas que favorezcan la reflexión por parte de los estudiantes sobre las temáticas tratadas en los artículos dan cuenta de ese propósito en la planificación.

\section{Discusión}

En términos generales, la propuesta evidencia falta de preguntas críticas que permitan discutir y reflexionar acerca del concepto de femicidio y las consecuencias que tiene en la sociedad en la que vivimos. Esta dificultad es reportada en Molfino y Ochoviet (2019) y es uno de los principales desafíos implicados en el diseño de actividades desde la perspectiva de la EMpJS. Es decir, cómo el profesor de matemática puede, a través de actividades, facilitar el ingreso al aula de temáticas socialmente relevantes y que estas sean discutidas por estudiantes y docentes, para tener la posibilidad de comprenderlas y, si se considera oportuno, intervenir para cambiarlas o contribuir a ello.

Observamos que una de las variables que puede haber incidido en la falta de preguntas críticas es la confusión evidenciada entre homicidio a mujeres y femicidio. Esto queda en evidencia cuando la profesora dice, en la fundamentación de la actividad propuesta, que “...no hay ninguna palabra que se utilice para referirse únicamente al homicidio de hombres". Como ya se explicó en la sección anterior, en Uruguay, el femicidio no es el homicidio de una mujer sino el homicidio de una mujer por el hecho de ser mujer. Son, entonces, nociones distintas, si bien vinculadas, y parecería que la profesora las confunde, pensando que femicidio es una denominación especial que se la da al homicidio de una mujer.

El artículo que selecciona para trabajar las partes 1, 2 y 3 de la actividad, no aborda datos estadísticos de femicidios sino de homicidios a mujeres $\mathrm{y}$, entre ellos, cuántos correspondieron a violencia doméstica. Quizás, para abordar la parte 3 en la que específicamente solicita a los estudiantes analizar la diferencia entre femicidio y homicidio, podría haber seleccionado una fuente que abordara directamente datos estadísticos referidos a femicidio, así como el análisis de la Ley 19538. En consecuencia, entendemos que una mejor comprensión de los conceptos involucrados hubiera permitido una mejor selección de las fuentes de información para tratar el tema y abordarlos desde la EMpJS. 


\section{LA VISIÓN DEL CONCEPTO DE FEMICIDIO EN EL PROFESORADO DE MATEMÁTICA. UN ESTUDIO DE CASO}

\section{CONCLUSIONES}

En suma, respecto a qué mensajes da la enseñanza de la matemática (What), observamos que la visión de femicidio que queda en evidencia a través de la secuencia didáctica elaborada por la profesora es la de que es sinónimo de homicidio a mujeres, sin tener en cuenta los aspectos sociales, jurídicos y afectivos vinculados a la figura penal femicidio. A su vez, tanto la secuencia como su fundamentación permiten identificar que la profesora considera que no es un crimen al que debiera otorgarse tanta atención, e incluso parecería sobredimensionado su tratamiento debido a que la tasa de homicidio a hombres es mayor que el de mujeres. De llevarse a cabo esta secuencia con estudiantes de profesorado podría generarse un mensaje erróneo sobre dicho crimen, distorsionando su concepción como injusticia social.

En este caso, la perspectiva de qué es el femicidio sería la de la profesora que elaboró la secuencia (Who). Nos cuestionamos, ¿es la perspectiva de esta profesora la que debe transmitirse en la matemática escolar? ¿Qué formación específica necesita el profesorado para que la perspectiva que se transmita a los estudiantes, en este caso futuros profesores, sea la de las leyes que definen la figura penal, y la de los movimientos sociales de mujeres y hombres que luchan en contra del femicidio? Situadas en la EMpJS, consideramos que esta última es una perspectiva adecuada a adoptar en la enseñanza de la matemática escolar para favorecer la comprensión del mundo a partir de ella: la de los grupos sociales que son vulnerados por las situaciones de injusticia.

Por último, proponemos una idea para lograr una vinculación entre los conceptos matemáticos y el mundo real en la matemática escolar (How). En la propuesta de la profesora podría plantearse estudiar el problema del femicidio a partir de los documentos que lo describen y documentos de diferentes colectivos de naturaleza social, y solicitar a los estudiantes que a partir de ello elaboren una presentación del tema, por ejemplo, mediante un artículo de prensa ficticio. Serían, entonces, los estudiantes quienes decidirían qué herramientas estadísticas podrían emplear para comparar y relacionar los diferentes datos que se encuentran en los documentos para sustentar el mensaje que quieren transmitir. Esta es una propuesta didáctica, entre otras, que se podría proponer con el fin de emplear los conceptos matemáticos para comprender mejor el mundo que nos rodea, en particular identificar las injusticias y, por qué no, luchar para superarlas. 

MATEMÁTICA. UN ESTUDIO DE CASO

\section{REFERENCIAS}

AGUIRRE, J. M. Privileging mathematics and equity in teacher education: Framework, counterresistance strategies, and reflections from a Latina mathematics educator. En: B. Greer, S. Mukhopadhyay, A. B. Powell y S. Nelson-Barber (eds.). Culturally responsive mathematics education. New York: Routledge, 2009, p. 295-319.

ÁlVAREZ, F.; MOLFINO, V.: PEREIRA, L.; SILVA, F. Alimentación saludable también para los adolescentes. En: BUENDÍA, G.; MOLFINO, V.; OCHOVIET, C. (Comp.), Estrechando lazos entre investigación y formación en Matemática Educativa. Volumen IV. Montevideo: CFE, 2017. p. 73-83. Disponible en: http://bit.ly/2IF0VF4. Acceso: 23 abr. 2019.

BENTANCORT, C.; BENTANCUR, Y.; BERTRAND, L.; FERNÁNDEZ, R.; IRAZUSTA, F.; IZQUIERDO, A.; PASTRO, M.; OCHOVIET, C. El teatro de títeres como recurso didáctico para la enseñanza de la matemática. En: BUENDÍA, G.; MOLFINO, V.; OCHOVIET, C. (Comp.), Estrechando lazos entre investigación y formación en Matemática Educativa. Volumen IV. Montevideo: CFE, 2017. p. 25-52. Disponible en: http://bit.ly/2IF0VF4. Acceso: 23 abr. 2019.

COLOMBO, A. Un Mundo Feliz: El Lugar de la Realidad en el Álgebra Lineal. En BUENDÍA, G.; MOLFINO, V.; OCHOVIET, C. (Comps.), Estrechando lazos entre investigación y formación en Matemática Educativa. Experiencias conjuntas de docentes y futuros docentes. Volumen IV. Montevideo: CFE, 2017. p. 53-69. Disponible en: http://bit.ly/2IF0VF4. Acceso: 23 abr. 2019.

DE LEÓN, V.; DELGADO, C.; MOLFINO, V.; SANTINI, B. Dime cuánto ganas y te diré dónde vives. En BUENDÍA, G.; MOLFINO, V.; OCHOVIET, C. (Comp.), Estrechando lazos entre investigación y formación en Matemática Educativa. Volumen III. Montevideo: CFE, 2016. p. 103-115. Disponible en: http://bit.ly/2USFgzy. Acceso: 23 abr. 2019.

DOLGAY, M.; OCHOVIET, C. Una historia de contadores. En: BUENDÍA, G.; MOLFINO, V.; OCHOVIET, C. (Comp.), Estrechando lazos entre investigación y formación en Matemática Educativa. Volumen III. CFE: Montevideo, 2016. p. 43-50. Disponible en: http://bit.ly/2USFgzy. Acceso: 23 abr. 2019.

ENSIGN, J. Helping teachers use students' home cultures in mathematics lessons: Developmental stages of becoming effective teachers of diverse students. En: Rodriguez, A. J.; KITCHEN, R. S. (Eds.), Preparing mathematics and science teachers for diverse classrooms: Promising strategies for transformative pedagogy. Mahwah, NJ: Lawrence Erlbaum Associates, 2005, p. 225-242.

FELTON, M. D.; KOESTLER, C. Questions and answers can mean something: Supporting critical reflection in mathematics education. En: FLESSNER, R.; MILLER, G. R.; PATRIZIO, K. M.; HORWITZ, J. R. (eds.), Agency through teacher education: Reflection, community, and learning. Lanham, MD: Rowman y Littlefield, 2012, p. 25-35. 


\section{LA VISIÓN DEL CONCEPTO DE FEMICIDIO EN EL PROFESORADO DE MATEMÁTICA. UN ESTUDIO DE CASO}

FELTON, M. D.; KOESTLER, C. Math is all around us and... we can use it to help us: Teacheragency in mathematics education through critical reflection. The New Educator, v. 11, n. 4, p. 260-276, nov. 2015. Disponible en: https://www.tandfonline.com/doi/abs/10.1080/1547688X.2015.1087745. Acceso: 23 febr. 2020.

FELTON, M. D.; SIMIC-MULLER, K.; MENÉNDEZ, J. M. Math isn't just numbers or algorithms: Mathematics for social justice in preservice K-8 content courses. En: Jacobsen, L. J.; Mistele, J.; Sriraman, B. (eds.), Mathematics teacher education in the public interest: Equity and social justice. Charlotte, NC: Information Age Publishingp, 2012, p. $231 \square 252$.

FELTON-KOESTLER, M. Mathematics education as sociopolitical: prospective teachers' views of the What, Who, and How. Journal of Mathematics Teacher Education, n. 20(1), p. 49-74, 2017.

GALLI, M.; MOLFINO, V.; MONTEGUI, E.; NÚÑEZ, I. Desnaturalizando lo socialmente establecido: una discusión de género. En: BUENDÍA, G.; MOLFINO, V.; OCHOVIET, C. (Comp.), Estrechando lazos entre investigación y formación en Matemática Educativa. Volumen IV. CFE: Montevideo, 2017. p. 85-96. Disponible en: http://bit.ly/2IF0VF4. Acceso: 23 abr. 2019.

GONZÁLEZ, L. Teaching mathematics for social justice: Reflections on a community of practice for urban high school mathematics teachers. Journal for Urban Mathematics Education, n. 2(1), p. 22-51, 2009.

GONZÁLEZ, S; GONZÁLEZ, V.; LEPRATTE, F.; MOLFINO, V.; VIERA, C. (2016). Un análisis crítico sobre la ganancia en el mundo del mercado. En BUENDÍA, G.; MOLFINO, V.; OCHOVIET, C. (Comp.), Estrechando lazos entre investigación y formación en Matemática Educativa. Volumen III. Montevideo: CFE, 2016. p. 85-101. Disponible en: http://bit.ly/2USFgzy. Acceso: 23 abr. 2019.

GUERRA, P.; LIM, W.; LÓPEZ, R. Math, social justice and prospective teachers in U.S.A. and Uruguay: learning together. En: CHRONAKI, A. (ed.), Mathematics Education and Life at Times of Crisis. Proceedings of the Ninth International Mathematics Education and Society Conference. University of Thessaly Pess, Volos, Greece, 2017. Disponible en: http://mes9.ece.uth.gr/portal/images/proceedings/MES9_Proceedings_low_Volume1.pdf. Acceso: 29 mar. 2019.

GUTSTEIN, E. Reading and writing the world with mathematics: Toward a pedagogy for social justice. New York: Routledge, 2006.

LA RED 21. 46 mujeres asesinadas en Uruguay entre enero y octubre de 2018, según datos oficiales. La Red 21, Montevideo, nov. 2018. Sección Violencia de Género. Disponible en: http://www.lr21.com.uy/comunidad/1386535-femicidios-homicidios-mujeres-violenciagenero-ministerio-interior-bonomi. Acceso: 23 febr. 2020.

LEIRÓS, L.; RAMÍREZ, V.; OCHOVIET, C. Leer el mundo a partir de recursos matemáticos: situaciones de injusticia social que afectan a niños y adolescentes. En: BUENDÍA, G.; MOLFINO, V.; OCHOVIET, C. (Comp.), Estrechando lazos entre Revista RBBA Revista Binacional Brasil Argentina 

MATEMÁTICA. UN ESTUDIO DE CASO

investigación y formación en Matemática Educativa. Volumen III. CFE: Montevideo, 2016. p. 51-64. Disponible en: http://bit.ly/2USFgzy. Acceso: 23 abr. 2019.

LLORENTE, M. Educar para la justicia social. Ponencia presentada en el Foro Mundial de Educación (Brasil), 2012. Disponible en: http://www.concejoeducativo.org/2012/educarpara-la-justicia-social/. Acceso: 29 mar. 2019.

LÓPEZ, R.; GUERRA, P. Enseñanza de la Matemática para la Justicia Social. Experiencia IFD de Pando -Universidad de Kennesaw, EEUU. En SEMUR, Actas del $7^{\circ}$ Congreso Uruguayo de Educación Matemática, 2017. p. 245-252. Disponible en http://semur.edu.uy/curem/actas/pdf/56.pdf. Acceso: 10 nov. 2017.

MINISTERIO DEL INTERIOR (MI). Femicidios íntimos en Uruguay. Homicidios a mujeres a manos de (ex) parejas. Montevideo: MI, 2017. Disponible en: https://www.minterior.gub.uy/genero/images/stories/Femicidios_Uruguay.pdf. Acceso: 23 febr. 2020.

MOLFINO, V.; PERDOMO, N.; RUIZ, X.; VILLA, S. Analfabetismo y afrodescendencia: ¿casualidad o causalidad? En: BUENDÍA, G.; MOLFINO, V.; OCHOVIET, C. (Comp.), Estrechando lazos entre investigación y formación en Matemática Educativa. Volumen IV. CFE: Montevideo, 2017. p. 97-111. Disponible en: http://bit.ly/2IF0VF4. Acceso: 23 abr. 2019.

MOLFINO, V.; OCHOVIET, C. Enseñanza de la matemática para la justicia social en cursos de postgraduación. Revista Latinoamericana de Matemática Educativa, Ciudad de México, v. 22, n. 2, p. 139-162, jul. 2019. Disponible en: http://relime.org/articulos/2202/201901b/. Acceso: 23 febr. 2020.

PARRA, V. Una propuesta didáctica para construcción de ciudadanía crítica a través del aprendizaje de la matemática. En: CONGRESO IBEROAMERICANO DE EDUCACIÓN MATEMÁTICA, VII, 2013, Montevideo. Actas... Montevideo: 2013. p. 3929-3937.

URUGUAY, Ley 17514 (2002). Ley de erradicación de la violencia doméstica. Disponible en: https://www.impo.com.uy/bases/leyes/17514-2002. Acceso: 23 febr. 2020.

URUGUAY, Ley 19538 (2017). Actos de discriminación y femicidio. Disponible en: https://legislativo.parlamento.gub.uy/temporales/docu7286979566912.htm. Acceso: febr. 2020.

RODRIGUEZ, A. J. Teachers' resistance to ideological and pedagogical change: Definitions, theoretical framework, and significance. En: RODRIGUEZ, A. J.; KITCHEN, R. S. (ed.), Preparing mathematics and science teachers for diverse classrooms: Promising strategies for transformative pedagogy. Mahwah, NJ: Lawrence Erlbaum Associates, 2005. p. $1-16$.

SCHAFFEL, V.; OCHOVIET, C. Consiguieron la paz en planilandia. En: BUENDÍA, G.; MOLFINO, V.; OCHOVIET, C. (Comp.), Estrechando lazos entre investigación y 


\section{LA VISIÓN DEL CONCEPTO DE FEMICIDIO EN EL PROFESORADO DE MATEMÁTICA. UN ESTUDIO DE CASO}

formación en Matemática Educativa. Volumen III. CFE: Montevideo, 2016. p. 29-42. Disponible en: http://bit.ly/2USFgzy. Acceso: 23 abr. 2019.

SKOVSMOSE, O. Alfabetismo matemático y globalización. En: VALERO, P.; SKOVSMOSE, O. (ed.), Educación matemática crítica. Una visión sociopolítica del aprendizaje y la enseñanza de las matemáticas. Bogotá: una empresa docente, 2012, p. 65105. Disponible en: http://funes.uniandes.edu.co/2003/1/Skovsmose2012Alfabetismo.pdf. Acceso: 29 mar. 2019.

STINSON, D.; BIDWELL, C.; POWELL, G. Critical pedagogy and teaching mathematics for social justice. The International Journal of Critical Pedagogy, n. 4(1), p. 76-94, 2012. Disponible en: http://libjournal.uncg.edu/ojs/index.php/ijcp/article/view/302/263. Acceso: 29 mar. 2019.

WRIGHT, P. Teacher researchers, mathematics classrooms and social justice. 2014. Presentación en BERA Conference 2014. Londres, 2014. Disponible en: http://mathssocialjustice.weebly.com/uploads/3/0/2/7/30279643/wright_2014_bera_paper.pdf. Acceso: 29 mar. 2019.

\section{Sobre as autoras}

Verónica Molfino Vigo es profesora de Matemática por el Instituto de Profesores Artigas (CFE, Montevideo) y Doctora en Matemática Educativa por Centro de Investigación en Ciencia Aplicada y Tecnología Avanzada (CICATA, IPN, México D. F.); profesora de Matemática y Didáctica de la Matemática en el profesorado de Matemática (CFE, Uruguay) y en el Diploma en Matemática (ANEP - UdelaR). Miembro del Sistema Nacional de Investigadores (Uruguay).

Correo electrónico: veromolfino@gmail.com

Cristina Ochoviet es Doctora en Matemática Educativa (CICATA-IPN, México). Magíster en Ciencias en Matemática Educativa (CICATA-IPN, México). Licenciada en Educación (UNQ, Argentina); profesora de Matemática (IPA, Uruguay); tiene Especialización en Lectura, Escritura y Educación (FLACSO, Argentina). Diploma en Constructivismo y Educación (FLACSO, Argentina). Se ha desempeñado como docente de Matemática en enseñanza media, como Profesora de Didáctica de la Matemática (IPA-CFE) y como investigadora (IPES-CFE). Su actividad de investigación se centra en la formación de profesores de Matemática para la enseñanza media y en el desarrollo del pensamiento algebraico.

Correo electrónico: cristinaochoviet@gmail.com 
Daniela Pagés. es Magíster en Ciencias en Matemática Educativa (CICATA-IPN, México); certificado en Enseñanza de la matemática en la formación de profesores (CFE-ANEP). Profesorado de Matemática (IPA-CFE). Se ha desempeñado como docente en la enseñanza media y en la formación docente dictando Didáctica y Matemática Básica en la especialidad Matemática (Profesora Semipresencial) y Matemática en la formación magisterial (IINN). Actualmente investiga las interacciones en la clase de práctica de los docentes en formación.

Correo electrónico: danielapages@ gmail.com 